\title{
Composição florística e fisionomia de floresta estacional semidecídua submontana na Chapada Diamantina, Bahia, Brasil ${ }^{1}$
}

\section{Floristic composition and physiognomy of a submontane seasonal semi-deciduous forest} on Chapada Diamantina, Bahia, Brazil

\author{
Ana Paula Lima do Couto $^{2}$, Lígia Silveira Funch ${ }^{3} \&$ Abel Augusto Conceição ${ }^{3}$
}

\begin{abstract}
Resumo
O estudo visou conhecer a composição florística e descrever a fisionomia de um trecho de floresta estacional semidecídua submontana e investigar as relações florísticas na Chapada Diamantina. A fisionomia foi caracterizada pelo perfil e hábitos das espécies. Foram identificadas 117 espécies de 85 gêneros em 49 famílias. Alguns táxons mais ricos em espécies são recorrentes em outras florestas estudadas no Brasil, destaque às famílias Fabaceae, Myrtaceae, Lauraceae e Apocynaceae, e aos gêneros Ocotea, Myrcia, Casearia e Inga. O dossel apresenta árvores de 10 a $16 \mathrm{~m}$ de altura, destacando-se Micropholis gardneriana e Pogonophora schomburgkiana, com emergentes até $26 \mathrm{~m}$ de altura. O subdossel é formado por indivíduos com 6 a $9 \mathrm{~m}$ de altura, representado pela grande maioria das espécies de árvores. O sub-bosque é formado, em sua maioria, por indivíduos jovens das espécies dos estratos superiores, espécies de Rubiaceae e Melastomataceae e Parodiolyra micrantha (Poaceae). Comparações com outras florestas revelaram táxons de maior constância relativa e maior riqueza do componente arbóreo na Chapada Diamantina: Myrtaceae, Fabaceae, Anacardiaceae, Apocynaceae, Sapotaceae, Simaroubaceae, Calyptranthes, Pouteria, Simarouba, Tapirira, Clusia, Miconia, Myrcia e Protium. O estudo revelou distinção entre a floresta estacional semidecídua submontana e as demais formações florestais da Chapada Diamantina, reforçando a necessidade de ampliação dos estudos florísticos e estruturais dessas florestas.
\end{abstract}

Palavras-chave: fitogeografia, similaridade, semi-árido.

\begin{abstract}
This study surveyed floristic composition and physiognomy of an area of submontane seasonal semi-deciduous forest and examine floristic relationships on the Chapada Diamantina. Angiosperm species were collected monthly in 2004 and from May 2006 to August 2007. Physiognomy was characterized by the profile and species' habit. A total of 117 species belonging to 85 genera and 49 families were identified. Some of the more species-rich taxa are recurrent in other forests in Brazil, especially the families Fabaceae, Myrtaceae, Lauraceae and Apocynaceae, and the genera Ocotea, Myrcia, Casearia and Inga. The canopy consists of trees 10-16m tall, such as Micropholis gardneriana (Sapotaceae) and Pogonophora schomburgkiana (Euphorbiaceae) with 26-meter-tall emergents. The sub-canopy is located approximately 6-9 m above the forest floor and contains most of the tree species. The understory is composed mostly of saplings of the species that form the upper strata, species of the families Rubiaceae and Melastomataceae and Parodiolyra micrantha (Poaceae). Comparing with other forests showed taxa previously found on the Chapada Diamantina, indicating greater consistency and greater richness of the tree component on the Chapada: Myrtaceae, Fabaceae, Anacardiaceae, Apocynaceae, Sapotaceae, Simaroubaceae, Calyptranthes, Pouteria, Simarouba, Tapirira, Clusia, Miconia, Myrcia and Protium. The study indicated a distinction between Submontane Seasonal Forest and other forest formations on the Chapada, showing the need for more floristic and structural studies there.
\end{abstract}

Key words: fitogeography, similarity, semiarid.

\footnotetext{
${ }^{1}$ Parte da dissertação de Mestrado em Botânica da primeira autora.

${ }^{2}$ Universidade Estadual do Sudoeste da Bahia, Depto. Estudos Básicos e Instrumentais, Área de Ciências Biológicas, Praça da Primavera 40, 45700-000, Itapetinga, BA. Autor para correspondência: aplcouto@yahoo.com.br

${ }^{3}$ Universidade Estadual de Feira de Santana, Depto. Ciências Biológicas, Curso de Pós-graduação em Botânica, BR 116 N km 03, Campus Universitário, 44031-460, Feira de Santana, BA.
} 


\section{Introdução}

O Brasil é um dos países com maior diversidade de ecossistemas florestais (Leitão-Filho 1987). Dentre estes ecossistemas, a floresta estacional insere-se no domínio da Mata Atlântica sensu lato, um dos ecossistemas brasileiros mais ameaçados (Morellato \& Haddad 2000; Oliveira-Filho \& Fontes 2000). Desta forma, a manutenção da diversidade biológica tornou-se importante alvo de várias iniciativas que buscam orientar a preservação de seus remanescentes (Diegues \& Arruda 2001; Oliveira-Filho et al. 2005a).

$\mathrm{O}$ incremento de pesquisas realizadas nas últimas décadas, principalmente na região Sudeste, propiciou considerável aumento do conhecimento sobre a Floresta Atlântica sensu lato, caracterizada como uma extensa e importante formação florestal extra-amazônica (Meira Neto et al. 1989; LeitãoFilho 1992). As primeiras avaliações de inventários florísticos sobre a Floresta Atlântica ocorreram em São Paulo (Salis et al. 1995; Torres et al. 1997; Ivanauskas et al. 2000; Scudeller et al. 2001; Oliveira 2006) e posteriormente em Minas Gerais (OliveiraFilho et al. 1994) e Rio de Janeiro (Peixoto et al. 2005); ou por regiões, como Sudeste (Oliveira-Filho \& Fontes 2000; Oliveira-Filho et al. 2005b) e Nordeste (Ferraz et al. 2004). Em maior amplitude geográfica, ressalta-se o trabalho de Siqueira (1994), com a análise de 63 áreas de Floresta Atlântica sensu stricto das Regiões Nordeste, Sudeste e Sul.

Na região central da Bahia situa-se a Chapada Diamantina, local de diversidade florística elevada, associada à presença de variados tipos de vegetação que incluem cerrados, florestas, caatingas e campos rupestres (Harley 1995; Funch et al. 2009). As florestas estacionais encontradas na borda leste da Chapada Diamantina foram caracterizadas através de seu componente arbóreo e de aspectos da topografia e solos, sendo identificadas florestas ciliares, florestas de encosta, florestas de planalto e florestas de grotão (Funch et al. 2005, 2008).

A formação florestal predominante nessa região é a Floresta Estacional Semidecidual Submontana (FESS) (Funch et al. 2005, 2008), que ocorre desde a Bahia, Espírito Santo, Rio de Janeiro, Minas Gerais, São Paulo, sudoeste do Paraná e sul do Mato Grosso do Sul, incluindo gêneros de plantas característicos, como Copaifera, Hymenaea, Dipteryx, Macherium, Aspidosperma, Casearia e Tabebuia (Veloso 1992; Rizzini 1997; Marangon et al. 2003; Couto 2008).
As florestas estacionais semideciduais submontanas (florestas de planalto em latossolos, a 400-600 m de altitude), objetos deste estudo, ocupavam no passado quase toda a borda oriental da Chapada Diamantina. Porém, nos últimos 20 anos foi observado o desaparecimento de uma expressiva porcentagem dessas florestas (Funch et al. 2005, 2008). Na tentativa de minimizar o avanço desse desmatamento, foram implantadas algumas unidades de conservação, como o Parque Nacional da Chapada Diamantina (PNCD) e a Área de Proteção Ambiental Marimbús Iraquara (Franca-Rocha et al. 2005; Funch et al. 2008; SEIA 2010).

No entanto, embora haja uma consciência crescente sobre a importância dos remanescentes florestais e da necessidade de conservá-los, é evidente que há uma relativa escassez de levantamentos na Chapada Diamantina. Os estudos ainda são pontuais na borda oriental da Chapada para florestas estacionais sub-montanas e montanas (Funch 1997; Stradmann 1997, 2000; Souza 2007; Funch et al. 2008; Ribeiro-Filho et al. 2009) e na borda ocidental para florestas estacionais montanas (Nascimento 2009).

Dessa forma, o presente trabalho visa conhecer a composição florística e descrever a fisionomia de um trecho de floresta estacional semidecidual submontana, bem como investigar as relações florísticas entre o componente arbóreo da área aqui estudada e de outras florestas estudadas na Chapada Diamantina.

\section{Materiais e Métodos}

\section{Área de estudo}

$\mathrm{O}$ trecho de floresta estudado localiza-se no município de Lençóis, no Parque Nacional da Chapada Diamantina, Bahia, Brasil. O acesso é realizado a partir da BR 242 (Salvador-Brasília) (Fig. 1).

A vegetação estudada é classificada como floresta estacional semidecidual submontana (FESS, segundo Veloso et al.1991), ocorrendo em relevo suave ondulado, em altitudes entre cerca de 400 a 600 m, sobre Latossolo Vermelho-Amarelo de textura argilosa e com pouca matéria orgânica (Jesus et al. 1985). Em geral, toda a região da Chapada Diamantina é predominantemente constituída de rochas metassedimentares com baixo grau de metamorfismo, de idade Proterozóica, frequentemente dobradas e afetadas por importantes exposições ígneas na sua parte ocidental (Jesus et al.1985). 


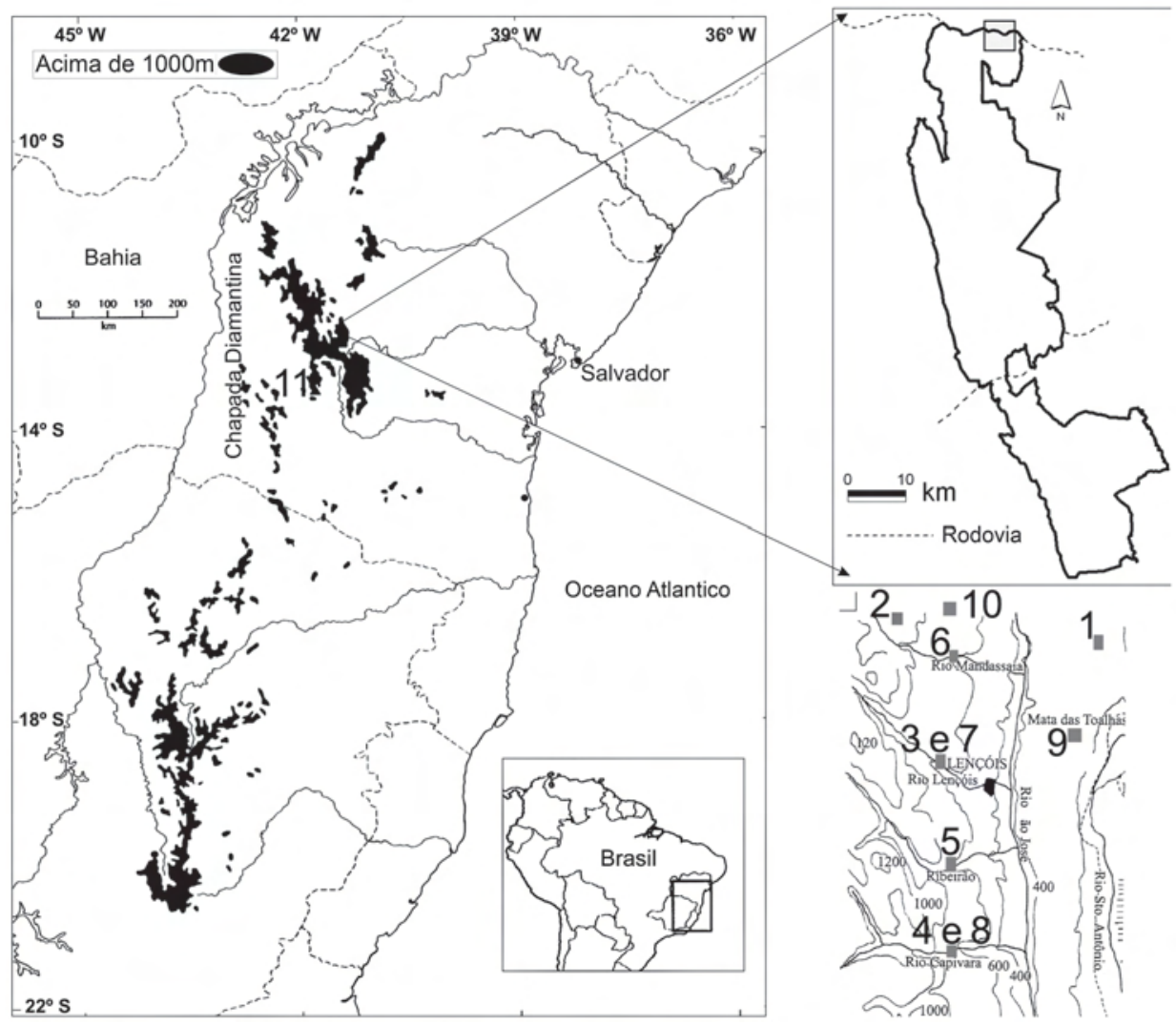

Figura 1 - Localização das florestas estudadas na Chapada Diamantina, Bahia, Brasil. As áreas estão numeradas conforme apresentado na Tabela 1.

Figure 1 - Localization of the studied forest in Chapada Diamantina, Bahia, Brazil. The indicated area comprehends the number as shown in Table 1.

Devido às condições especiais de altitude, $\mathrm{o}$ clima da região de Lençóis é mesotérmico do tipo Cwb, com máximo de chuvas no verão (novembro, dezembro e janeiro) e um máximo secundário em março-abril, com verão chuvoso e inverno seco (RADAMBRASIL 1981). A pluviosidade varia entre $35 \mathrm{~mm}$ (julho e agosto) a $184 \mathrm{~mm}$ (dezembro), com precipitação média excedendo $100 \mathrm{~mm}$ durante a estação chuvosa. As temperaturas médias mensais oscilam durante o ano, variando em torno de $18^{\circ} \mathrm{C}$ durante o inverno (abril-setembro) e excedendo $22^{\circ} \mathrm{C}$ nos meses mais quentes (outubro-fevereiro) (Funch et al. 2002).

\section{Levantamento florístico}

As coletas foram realizadas através de caminhadas aleatórias em 2004 e no período de maio/2006 a agosto/2007. Durante esse período foram feitas visitas mensais na busca de ramos férteis de angiospermas. Espécies cujos indivíduos não foram observados em estádio reprodutivo foram registradas por meio de amostras estéreis de espécies amostradas no estudo fitossociológico realizado por Couto (2008). As espécies foram classificadas quanto ao hábito de acordo com os critérios adotados por Ribeiro et al. (1999).

O material coletado foi processado segundo a metodologia de Mori et al. (1989), herborizado e incluído no Herbário da Universidade Estadual de Feira de Santana (HUEFS). As exsicatas estéreis foram armazenadas na coleção permanente do Laboratório de Flora e Vegetação/UEFS, como testemunhos. A identificação do material foi realizada utilizando-se bibliografia especializada, comparação com exemplares depositados no HUEFS, além de consulta direta a especialistas. A classificação taxonômica baseou-se no sistema APGII (2003). 


\section{Diagrama de perfil da vegetação}

Foi elaborado um diagrama de perfil (Kershaw \& Looney 1985), a fim de visualizar a distribuição horizontal e vertical e a distribuição das formas de vida presentes na vegetação estudada (OliveiraFilho \& Fluminhan-Filho 1999). O perfil representou uma faixa com $30 \mathrm{~m}$ de comprimento e $10 \mathrm{~m}$ de largura, situada aleatoriamente a 40 metros da borda da floresta e delimitada por trena e corda, onde todos os indivíduos foram identificados e medidos.

\section{Similaridade florística}

As relações florísticas entre as florestas estudadas na Chapada Diamantina foram avaliadas pela similaridade de Jaccard (Müeller-Dombois \& Ellenberg 1974). Devido ao fato da maioria dos estudos terem sido direcionados às árvores, a similaridade calculada restringiu-se ao componente arbóreo, utilizando-se uma matriz de presença e ausência com 266 espécies arbóreas identificadas em nível específico incluídas no presente estudo e nos demais levantamentos florísticos realizados na Chapada Diamantina (Tab.1; Fig.1). A maioria dos dados florísticos comparados foi obtida através de parcelas $10 \times 10 \mathrm{~m}$ contínuas ou aleatórias (presente estudo), exceto Funch (1997), Funch et al. (2008) e Nascimento (2009).

Um quadro comparativo entre as florestas foi elaborado considerando-se as famílias mais ricas em espécies de cada área, incluindo apenas os táxons identificados em nível de espécie. Nas listagens florísticas, as famílias estão tratadas segundo APGII (2003). Foram analisadas famílias e gêneros com as maiores constâncias relativas e proporções de espécies nos diversos levantamentos. A constância relativa de um táxon $(\mathrm{CRt})$ considera o número de levantamentos com presença do táxon $(\mathrm{Pt})$ em relação ao total de levantamentos $(\mathrm{T}): \mathrm{CRt}=$ 100Pt/T (Müeller-Dombois \& Ellenberg 1974; Yamamoto et al. 2005).

Tabela 1 - Relação das florestas da Chapada Diamantina, Bahia, Brasil utilizadas na análise de similaridade florística. 1. Presente estudo; 2. Mata de Encosta Serra da Bacia (Souza 2007); 3. Mata Ciliar Rio Lençóis (Funch 1997); 4. Mata Ciliar Rio Capivara (Stradmann 2000); 5. Mata Ciliar Rio Ribeirão (Stradmann 1997); 6. Mata Ciliar Rio Mandassaia (Ribeiro-Filho et al. 2009); 7. Mata de Encosta Rio Lençóis (Funch 1997); 8. Mata de Encosta Rio Capivara (Stradmann 2000); 9. Mata de Planalto (Funch et al. 2008); 10. Mata de grotão (Funch et al. 2008); 11. Mata Montana (Nascimento 2009). FEM = Floresta Estacional Montana; FES = Floresta Estacional Submontana; LVA = Latossolo Vermelho-Amarelo; RE = Riqueza específica; EA = Espécies arbóreas.

Table 1 - List of the forests of the Chapada Diamantina, Bahia, Brazil used in the analysis of floristic similarity. 1. Present study, 2. Montane forests in the Bacia mountain chain (Souza 2007); 3. Ciliary forests along the Lençóis River (Funch 1997); 4. Ciliary forests along the Capivara River (Stradmann 2000); 5. Ciliary forests Ribeirão River (Stradmann 1997); 6. Ciliary forests along the Mandassaia River (Ribeiro-Filho et al. 2009); 7. Montane forests besides of the Lençóis River Valley (Funch 1997); 8. Montane forests besides of the Capivara River Valley (Stradmann 2000); 9. Tableland forest (Funch et al. 2008); 10. Crevice forest (Funch et al. 2008); 11. Montane forest (Nascimento 2009). FEM = Montane Seasonal Forest; FES = Submontane Seasonal Forest; LVA $=$ Latosols Red-Yellow; RE $=$ Species Richness; EA $=$ Arboreal Species.

\begin{tabular}{lclllllc}
\hline Área & Fisionomia & Deciduidade & \multicolumn{1}{c}{ Coordenadas } & Altitude $(\mathbf{m})$ & Solo & Clima & RE/EA \\
\hline 1 & FES & Semidecidual & $12^{\circ} 28^{\prime} \mathrm{S}-41^{\circ} 23^{\prime} \mathrm{W}$ & $500-600$ & LVA & Cwb & $117 / 88$ \\
2 & FEM & Perenifólia & $12^{\circ} 27^{\prime} \mathrm{S}-41^{\circ} 28^{\prime} \mathrm{W}$ & 1.100 & Litólico & Cwb & $94 / 71$ \\
3 & FES & Perenifólia & $12^{\circ} 33^{\prime} \mathrm{S}-41^{\circ} 24^{\prime} \mathrm{W}$ & $450-500$ & Litólico & Cwb & 81 \\
4 & FES & Perenifólia & $12^{\circ} 38^{\prime} \mathrm{S}-41^{\circ} 23^{\prime} \mathrm{W}$ & 600 & Litólico & Aw & $142 / 64$ \\
5 & FES & Perenifólia & $12^{\circ} 35^{\prime} \mathrm{S}-41^{\circ} 23^{\prime} \mathrm{W}$ & 800 & Litólico & Cwb & 38 \\
6 & FES & Perenifólia & $12^{\circ} 33^{\prime} \mathrm{S}-41^{\circ} 25^{\prime} \mathrm{W}$ & 800 & Litólico & Cwb & 42 \\
7 & FEM & Perenifólia & $12^{\circ} 33^{\prime} \mathrm{S}-41^{\circ} 24^{\prime} \mathrm{W}$ & $600-700$ & Litólico & Cwb & 55 \\
8 & FEM & Semidecidual & $12^{\circ} 38^{\prime} \mathrm{S}-41^{\circ} 23^{\prime} \mathrm{W}$ & $950-1.000$ & Litólico & Aw & $116 / 69$ \\
9 & FES & Semidecidual & $12^{\circ} 30^{\prime} \mathrm{S}-41^{\circ} 21^{\prime} \mathrm{W}$ & $400-500$ & LVA & Cwb & $45 / 45$ \\
10 & FEM & Perenifólia & $12^{\circ} 27^{\prime} \mathrm{S}-41^{\circ} 25^{\prime} \mathrm{W}$ & $1.000-1.200$ & Litólico & Cwb & $39 / 39$ \\
11 & FEM & Perenifólia & $13^{\circ} 31^{\prime} \mathrm{S}-41^{\circ} 58^{\prime} \mathrm{W}$ & $1.350-1750$ & Litólico & Cwb & $117 / 117$ \\
\hline
\end{tabular}




\section{Resultados}

\section{Diagrama de perfil da vegetação}

A vegetação analisada apresenta diferentes estratos, como pode ser observado no perfil (Fig. 2). Esta floresta apresenta um estrato superior não contínuo constituído por um dossel formado por árvores que variam de 10 a $16 \mathrm{~m}$ de altura, onde se destacam Micropholis gardneriana [numeração no perfil (NP) 1], Pogonophora schomburgkiana (NP 15), Tapirira guianensis (NP 30), Diospyros sericea (NP 33) e Pouteria ramiflora (NP 3); e emergentes que alcançam 18 a $26 \mathrm{~m}$, como Protium heptaphyllum (NP 6), Maprounea guianensis (NP 23), Hirtella glandulosa (NP 10) e Aspidosperma discolor (NP 37). Abaixo deste dossel, situa-se um estrato arbóreo contínuo, com cerca de 6 a $9 \mathrm{~m}$ de altura, representado pela grande maioria das árvores.

O sub-bosque é bem iluminado formado, em sua maioria, por indivíduos jovens das espécies dos estratos superiores e espécies de Rubiaceae e Melastomataceae. A espécie Parodiolyra micrantha (Poaceae) foi presente em grande parte da área amostrada. Trepadeiras são abundantes, destacando-se Ruellia affinis, Coccoloba striata, Davilla rugosa, Smilax sp. e Paullinia sp. Epífitas são raras, tendo-se registrado a presença de Campylocentrum micranthum, Vanilla sp. e Notyla sp. A lista completa das espécies coletadas e representadas no perfil pode ser visualizada na Tabela 2 .

\section{Levantamento florístico}

Foram registradas 117 espécies e 85 gêneros, distribuídos em 49 famílias de angiospermas (Tab. 2), sendo 88 espécies arbóreas (75\%), 15 trepadeiras (13\%), seis arbustivas (5\%), cinco herbáceas (4\%) e três epífitas (3\%), das quais 23 foram identificadas apenas em nível de gênero e duas em nível de família.

As famílias com maior riqueza de espécies foram Fabaceae com 13 espécies $(11,9 \%)$, Myrtaceae e Lauraceae com 10 espécies cada $(8,6 \%)$, Apocynaceae com sete espécies $(6 \%)$,

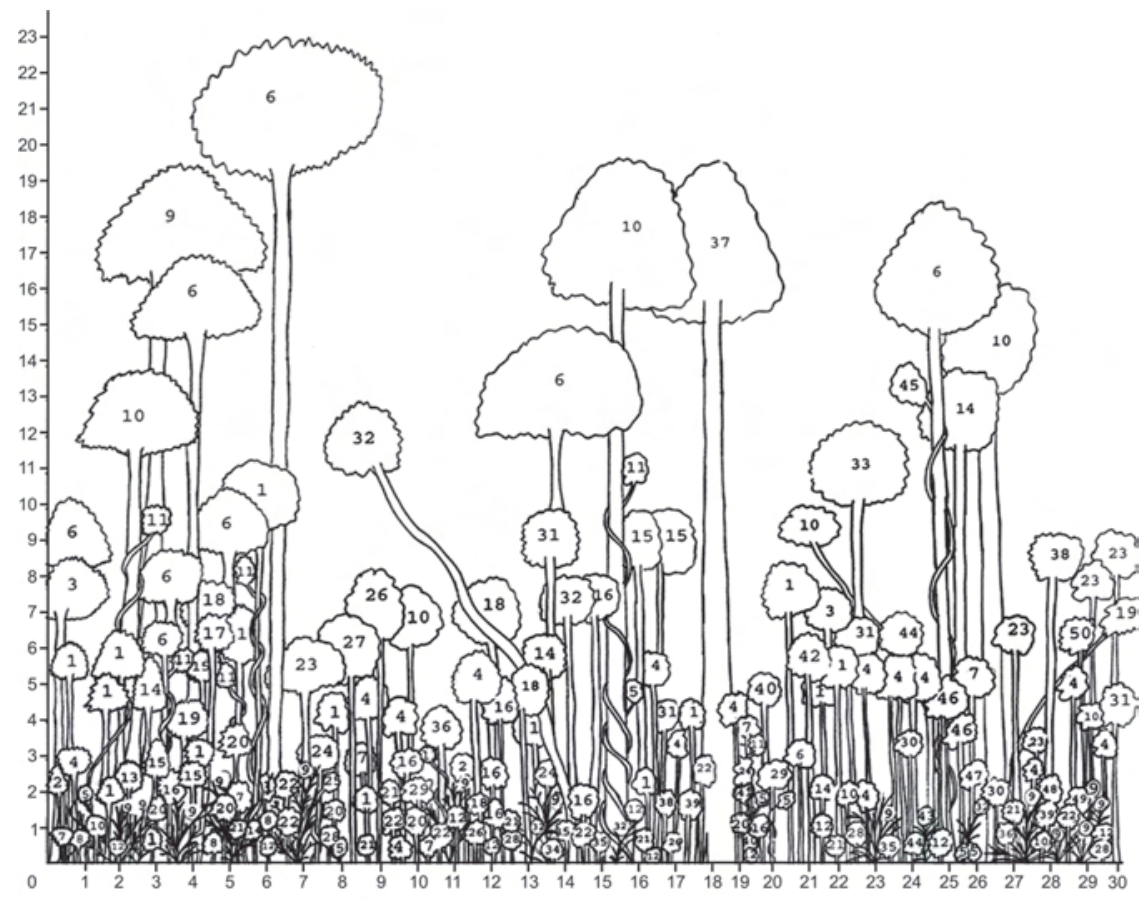

Figura 2 - Diagrama de Perfil de um trecho de $10 \times 30 \mathrm{~m}$ de Floresta Estacional Semidecidual Submontana no Parque Nacional da Chapada Diamantina, Lençóis, Bahia, com indivíduos numerados conforme espécies listadas na Tabela 2. Figure 2 - Profile diagram of an area of $10 \times 30 \mathrm{~m}$ Submontane Semideciduous Seasonal Forest in the Chapada Diamantina National Park, Lençóis, Bahia, Brazil, with individuals numbered as listed species in Table 2. 
Chrysobalanaceae e Rubiaceae com seis cada $(5,1 \%)$ e Euphorbiaceae com cinco espécies $(4,3 \%)$ (Tab. 3). O somatório da riqueza destas sete famílias representa $48,7 \%$ do total das espécies levantadas neste estudo. As 60 espécies restantes distribuíram-se em 42 famílias.

Os gêneros com maior riqueza de espécies foram Ocotea com sete espécies; Myrcia com quatro espécies; Casearia, Inga, Byrsonima, Miconia e Eugenia com três espécies cada. Onze gêneros foram representados por duas espécies e 68 gêneros por apenas uma.

\section{Similaridade florística}

Ao analisar os levantamentos florísticos na região da Chapada Diamantina, foram registradas 266 espécies arbóreas e 153 gêneros, distribuídos em 69 famílias de angiospermas. As famílias com maior riqueza específica apresentaram valores de constância relativa acima de $80 \%$, sendo máximo para as famílias Anacardiaceae, Apocynaceae, Fabaceae, Myrtaceae, Sapotaceae e Simaroubaceae, nas 11 áreas comparadas (Tab. 4).

Por meio do dendrograma de similaridade, com correlação cofenética de 0,89 , é possível distinguir dois grupos com similaridade de Jaccard a partir de $25 \%$ (Fig. 3). Um grupo (B) incluindo as áreas 5, 6, 10 e 11 respectivamente a Mata Ciliar do Rio Ribeirão (FES); a Mata Ciliar do Rio Mandassaia (FES), a Mata de Grotão (FEM) e a Mata de Rio de Contas/Rio do Pires (FEM); e o grupo (A) com sete, este último incluindo o par mais similar (60\%) formado pela Mata de Encosta do Rio Lençóis (FEM) e a Mata de Planalto (FES). O presente estudo apresenta $37 \%$ de similaridade com o grupo $\mathrm{C}$ formado por cinco áreas $(4,8,7,93)$, que juntos formam um grupo $45 \%$ similar.

Tabela 2 - Famílias e espécies ocorrentes em um trecho de Floresta Estacional Semidecidual Submontana no Parque Nacional da Chapada Diamantina, Lençóis, Bahia, Brasil, com respectivos hábitos, materiais testemunhos e numeração indicada no diagrama de perfil. MV = material vegetativo; * = espécies referidas apenas no presente estudo em comparação com outras listagens da Chapada Diamantina; $\mathrm{X}=$ ausente no diagrama de perfil.

Table 2 - Plant families and species occurring in the analyzed area of the Submontane Seasonal Forest in the Chapada Diamantina National Park, Lençóis, Bahia, Brazil. With their habits, specimen and numbering shown in the profile's diagram. MV = vegetative material, * = species listed only in the present study compared to other listings of Chapada Diamantina, $\mathrm{X}=$ absent in the profile's diagram.

\begin{tabular}{|c|c|c|c|}
\hline Família/Espécie & Hábito & $\begin{array}{l}\text { Testemunho } \\
\text { HUEFS }\end{array}$ & $\begin{array}{l}\text { Numeração } \\
\text { indicada no } \\
\text { perfil }\end{array}$ \\
\hline \multicolumn{4}{|l|}{ ACANTHACEAE } \\
\hline Ruellia affinis (Nees) Lindau & trepadeira & 120568 & $X$ \\
\hline \multicolumn{4}{|l|}{ ANACARDIACEAE } \\
\hline Tapirira guianensis Aubl. & árvore & 120582 & 30 \\
\hline \multicolumn{4}{|l|}{ ANNONACEAE } \\
\hline Guatteria oligocarpa Mart.* & árvore & 120563 & 48 \\
\hline Xylopia sp. & árvore & MV & $\mathrm{X}$ \\
\hline \multicolumn{4}{|l|}{ APOCYNACEAE } \\
\hline Aspidosperma discolor A. DC. & árvore & MV & 37 \\
\hline Blepharodon nitidum (Vell.) J. F. Macbr & trepadeira & 120591 & $X$ \\
\hline Himatanthus bracteatus (A.DC.) Woodson & árvore & 120542 & 14 \\
\hline Mandevilla scabra (Roem. \& Shutt.) K. Schum. & trepadeira & 120589 & $\mathrm{X}$ \\
\hline Odontadenia lutea (Vell.) Markgr. & trepadeira & 120534 & $\mathrm{X}$ \\
\hline Odontadenia sp. & trepadeira & MV & 25 \\
\hline Temnademia violacea (Vell.) Miers & trepadeira & 120590 & $\mathrm{X}$ \\
\hline \multicolumn{4}{|l|}{ ARALIACEAE } \\
\hline Schefflera sp. & árvore & MV & $X$ \\
\hline
\end{tabular}




\begin{tabular}{llll}
\hline Família/Espécie & Hábito & $\begin{array}{l}\text { Testemunho } \\
\text { HUEFS }\end{array}$ & $\begin{array}{l}\text { Numeração } \\
\text { indicada no } \\
\text { perfil }\end{array}$ \\
\hline
\end{tabular}

\section{ASTERACEAE}

Mikania lindbergii Baker in Mart.

\begin{tabular}{|c|c|c|}
\hline trepadeira & 120535 & $X$ \\
\hline trepadeira & 124622 & $\mathrm{X}$ \\
\hline árvore & MV & $\mathrm{X}$ \\
\hline árvore & 120540 & 6 \\
\hline árvore & MV & 27 \\
\hline árvore & MV & 8 \\
\hline árvore & MV & $X$ \\
\hline árvore & 120549 & 10 \\
\hline árvore & 120574 & 17 \\
\hline árvore & MV & $\mathrm{X}$ \\
\hline árvore & MV & 42 \\
\hline árvore & 120576 & 36 \\
\hline árvore & MV & $\mathrm{X}$ \\
\hline erva & 120532 & 43 \\
\hline erva & MV & 32 \\
\hline trepadeira & 120537 & 35 \\
\hline árvore & 120552 & 33 \\
\hline árvore & MV & $\mathrm{X}$ \\
\hline árvore & MV & $\mathrm{X}$ \\
\hline árvore & 120551 & 23 \\
\hline árvore & MV & 24 \\
\hline árvore & 120556 & 15 \\
\hline árvore & MV & X \\
\hline árvore & MV & 49 \\
\hline arbusto & MV & $\mathrm{X}$ \\
\hline trepadeira & MV & 45 \\
\hline árvore & 120575 & $X$ \\
\hline árvore & MV & 7 \\
\hline árvore & MV & $\mathrm{X}$ \\
\hline árvore & MV & 38 \\
\hline árvore & MV & 34 \\
\hline
\end{tabular}

BIGNONIACEAE

Pyrostegia venusta (Ker-Gawler) Miers

BORAGINACEAE

Cordia bicolor A. DC.*

BURSERACEAE

Protium heptaphyllum (Aubl.) Marchand

CELASTRACEAE

Maytenus robusta Reissek

CHRYSOBALANACEAE

Couepia cf. impressa Prance

Couepia sp.

Hirtella glandulosa Spreng.

Hirtella gracilipes (Hook. f.) Prance

Licania kunthiana Hook.f.

Licania sp.

CLUSIACEAE

Clusia nemorosa G. Mey

COMBRETACEAE

Buchenavia capitata (Vahl.) Eichl

CYPERACEAE

Rhynchospora $\mathrm{sp.}$

Cyperaceae Indeterminada

DILLENIACEAE

Davilla rugosa Poir.

EBENACEAE

Diospyros sericea A.DC.

EUPHORBIACEAE

Aparisthmium cordatum (A. Juss.) Baill

Chaetocarpus echinocarpus (Baill.) Ducke

Maprounea guianensis Aubl.

Pera glabrata (Schott) Poepp.ex Baill.

Pogonophora schomburgkiana Miers ex Benth.

FABACEAE

Anadenanthera colubrina var. colubrina (Griseb.) Altsch.

Andira fraxinifolia Benth.

Bauhinia funchiana A.Vaz

Bauhinia sp.

Copaifera langsdorffii Desf.

Hymenolobium janeirense var. stipulatum (N. Mattos) Lima

Inga laurina (Sw.) Willd.

Inga thibaudiana D.C

Inga sp.1

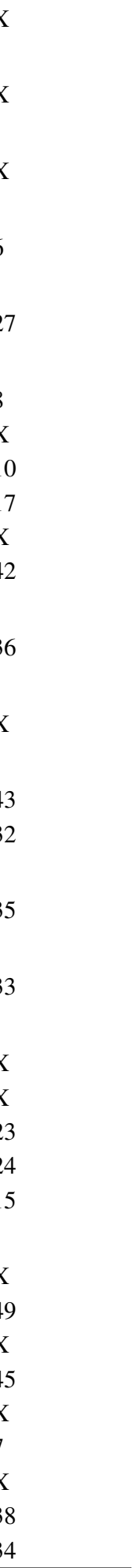




\begin{tabular}{|c|c|c|c|}
\hline Família/Espécie & Hábito & $\begin{array}{l}\text { Testemunho } \\
\text { HUEFS }\end{array}$ & $\begin{array}{l}\text { Numeração } \\
\text { indicada no } \\
\text { perfil }\end{array}$ \\
\hline Senna macranthera (Collad.) H.S. Irwin \& Barneby & árvore & 110075 & $\mathrm{X}$ \\
\hline Swartzia apetala Raddi & árvore & MV & $\mathrm{X}$ \\
\hline Swartzia bahiensis Cowan & árvore & 120547 & $\mathrm{X}$ \\
\hline Fabaceae indeterminada 1 & arbusto & MV & 20 \\
\hline \multicolumn{4}{|l|}{ HYPERICACEAE } \\
\hline Vismia guianensis (Aubl.) Choisy & árvore & 120583 & $\mathrm{X}$ \\
\hline \multicolumn{4}{|l|}{ ICACINACEAE } \\
\hline Emmotum nitens (Benth.) Miers & árvore & 120538 & $\mathrm{X}$ \\
\hline \multicolumn{4}{|l|}{ LACISTEMACEAE } \\
\hline Lacistema robustum Schinizl. & árvore & 120555 & $\mathrm{X}$ \\
\hline \multicolumn{4}{|l|}{ LAURACEAE } \\
\hline Aiouea guianensis Aubl. & árvore & MV & 19 \\
\hline Сіпnатотит sp. & árvore & MV & $\mathrm{X}$ \\
\hline Nectandra membranacea Griseb. & árvore & MV & 31 \\
\hline Ocotea corymbosa (Meisn.) Mez * & árvore & MV & $\mathrm{X}$ \\
\hline Ocotea diospyrifolia (Meisn.) Mez * & árvore & MV & 50 \\
\hline Ocotea glomerata $(\mathrm{Ness}) \mathrm{Mez} *$ & árvore & 120580 & $\mathrm{X}$ \\
\hline Ocotea nitida (Meisn.) Rohwer * & árvore & 96130 & 18 \\
\hline Ocotea sp.1 & árvore & MV & 29 \\
\hline Ocotea sp.2 & árvore & MV & $\mathrm{X}$ \\
\hline Ocotea sp.3 & árvore & MV & 47 \\
\hline \multicolumn{4}{|l|}{ LECYTHIDACEAE } \\
\hline Eschweilera tetrapetala Mori & árvore & $96124 / 110082$ & $\mathrm{X}$ \\
\hline \multicolumn{4}{|l|}{ LOGANIACEAE } \\
\hline Antonia ovata Pohl & árvore & 120541 & $\mathrm{X}$ \\
\hline \multicolumn{4}{|l|}{ MALPIGHIACEAE } \\
\hline Byrsonima crassifolia $(\mathrm{L}$.) Kunth * & árvore & MV & $\mathrm{X}$ \\
\hline Byrsonima sericea DC. & árvore & 120554 & $\mathrm{X}$ \\
\hline Byrsonima sp. & árvore & MV & $\mathrm{X}$ \\
\hline \multicolumn{4}{|l|}{ MALVACEAE } \\
\hline Eriotheca gracilipes (K. Schum.) A. Robyns * & árvore & MV & $\mathrm{X}$ \\
\hline \multicolumn{4}{|l|}{ MARANTHACEAE } \\
\hline Monotagma plurispicatum (Koern.) Schum & erva & 120546 & $\mathrm{X}$ \\
\hline \multicolumn{4}{|l|}{ MELASTOMATACEAE } \\
\hline Miconia holosericea $(\mathrm{L}) \mathrm{DC}$ & árvore & 120559 & 4 \\
\hline Miconia rimalis Naudin. * & árvore & $120539 / 120581$ & 26 \\
\hline Miconia sp. & árvore & MV & 44 \\
\hline \multicolumn{4}{|l|}{ MELIACEAE } \\
\hline Cabralea canjerana (Vell.) Mart. * & árvore & MC & $\mathrm{X}$ \\
\hline \multicolumn{4}{|l|}{ MYRSINACEAE } \\
\hline Myrsine umbellata Mart. & árvore & 110076 & $\mathrm{X}$ \\
\hline Myrsine guianensis (Aubl.) Kuntze & árvore & 96127 & $\mathrm{X}$ \\
\hline
\end{tabular}




\begin{tabular}{llll}
\hline Família/Espécie & Hábito & $\begin{array}{l}\text { Testemunho } \\
\text { HUEFS }\end{array}$ & $\begin{array}{l}\text { Numeração } \\
\text { indicada no } \\
\text { perfil }\end{array}$ \\
\hline
\end{tabular}

\section{MYRTACEAE}

Calyptranthes lucida DC.

Eugenia laxa DC. *

Eugenia platyclada O.Berg

Eugenia sp.

Myrcia detergens Miq.

Myrcia fallax (Rich.) DC. *

Myrcia obovata (Berg) Niedenzu *

Myrcia rostrata DC.

Myrciaria dubia (Kunth) Mc Vaugh *

Psidium brownianum DC.

OLACACEAE

Heisteria perianthomega (Vell.) Sleumer

Schoepfia obliquifolia Turcz.

ORCHIDACEAE

Campylocentrum micranthum Rolfe

Notyla sp.

Vanilla sp.

\begin{tabular}{|c|c|c|}
\hline árvore & MV & $\mathrm{X}$ \\
\hline árvore & MV & $\mathrm{X}$ \\
\hline árvore & 120592 & $X$ \\
\hline árvore & MV & $\mathrm{X}$ \\
\hline árvore & 96121 & 46 \\
\hline árvore & MV & $\mathrm{X}$ \\
\hline árvore & 120577 & $\mathrm{X}$ \\
\hline árvore & 120553 & 22 \\
\hline árvore & 96112 & $X$ \\
\hline árvore & MV & $\mathrm{X}$ \\
\hline árvore & MV & 2 \\
\hline árvore & MV & $X$ \\
\hline epífita & MV & $\mathrm{X}$ \\
\hline epífita & MV & $\mathrm{X}$ \\
\hline epífita & MV & $\mathrm{X}$ \\
\hline erva & 120569 & 9 \\
\hline erva & 96114 & $X$ \\
\hline trepadeira & 120562 & $\mathrm{X}$ \\
\hline árvore & MV & 13 \\
\hline árvore & 120531 & $\mathrm{X}$ \\
\hline trepadeira & 120579 & 11 \\
\hline árvore & 110081 & $\mathrm{X}$ \\
\hline arbusto & 120561 & 28 \\
\hline arbusto & 96115 & $\mathrm{X}$ \\
\hline arbusto & 120530 & 12 \\
\hline árvore & MV & $\mathrm{X}$ \\
\hline árvore & MV & 16 \\
\hline árvore & 120573 & $\mathrm{X}$ \\
\hline árvore & 120571 & $\mathrm{X}$ \\
\hline árvore & MV & $\mathrm{X}$ \\
\hline trepadeira & 120588 & 5 \\
\hline árvore & 120578 & 1 \\
\hline árvore & MV & 3 \\
\hline árvore & MV & 40 \\
\hline
\end{tabular}

POACEAE

Parodiolyra micrantha (Kunth) Davidse \& Zuloaga

POLYGALACEAE

Polygala oxyplylla DC

Coccoloba striata Benth.

PROTEACEAE

Euplassa sp.

RUBIACEAE

Amaioua guianensis Aubl.

Malanea sp.

Malopanthera paniculata Turcz. *

Palicourea marcgravii A. St.-Hil.

Psychotria hoffmansegianna (Roem. \& Schult.) Muell. Arg.

Psychotria barbiflora DC.

\section{RUTACEAE}

Hortia arborea Engl.

\section{SALICACEAE}

Casearia arborea (Rich) Urb.

Casearia commersoniana Cambess.

Casearia decandra Jacq. (Rich.) Urb. *

SAPINDACEAE

Cupania cf oblongifolia Mart.

Paullinia sp.

\section{SAPOTACEAE}

Micropholis gardneriana (DC.) Pierre

Pouteria ramiflora (Mart.) Radlk.

Pouteria torta (Mart.) Radlk.

árvore

MV 


\begin{tabular}{|c|c|c|c|}
\hline Família/Espécie & Hábito & $\begin{array}{l}\text { Testemunho } \\
\text { HUEFS }\end{array}$ & $\begin{array}{l}\text { Numeração } \\
\text { indicada no } \\
\text { perfil }\end{array}$ \\
\hline \multicolumn{4}{|l|}{ SIMAROUBACEAE } \\
\hline Simarouba amara Aubl. & árvore & MV & $\mathrm{X}$ \\
\hline \multicolumn{4}{|l|}{ SMILACACEAE } \\
\hline Smilax sp.1 & trepadeira & 120566 & $\mathrm{X}$ \\
\hline Smilax sp.2 & trepadeira & MV & 41 \\
\hline \multicolumn{4}{|l|}{ SIPARUNACEAE } \\
\hline Siparuna guianensis Aubl. & árvore & MV & $\mathrm{X}$ \\
\hline \multicolumn{4}{|l|}{ SYMPLOCACEAE } \\
\hline Symplocos nitens (Pohl.) Benth. * & árvore & 120572 & $\mathrm{X}$ \\
\hline \multicolumn{4}{|l|}{ TRIGONIACEAE } \\
\hline Trigonia eriosperma (Lam.) Fromm \& E. Santos & árvore & 120584 & $\mathrm{X}$ \\
\hline \multicolumn{4}{|l|}{ TURNERACEAE } \\
\hline Turnera cearensis Urb. & árvore & 120587 & $\mathrm{X}$ \\
\hline \multicolumn{4}{|l|}{ VOCHYSIACEAE } \\
\hline Qualea sp. & árvore & MV & 21 \\
\hline Vochysia tucanorum Mart. * & árvore & 120585 & $\mathrm{X}$ \\
\hline Vochysia sp. & arbusto & MV & 39 \\
\hline
\end{tabular}

Tabela 3 - Número de espécies por família, dentre as famílias mais ricas, nas florestas da Chapada Diamantina, Bahia, Brasil. O número entre parênteses refere-se à ordem de predominância da família nos respectivos estudos. 1. Presente estudo; 2 . Mata de Encosta Serra da Bacia; 3. Mata Ciliar Rio Lençóis; 4. Mata Ciliar Rio Capivara; 5. Mata Ciliar Rio Ribeirão; 6. Mata Ciliar Rio Mandassaia; 7. Mata de Encosta Rio Lençóis; 8. Mata de Encosta Rio Capivara; 9. Mata de Planalto; 10. Mata de grotão; 11. Mata Montana. Myrt $=$ Myrtaceae; Lau $=$ Lauraceae; $\mathrm{Fab}=$ Fabaceae Chrys $=$ Chrysobalanaceae; Euph $=$ Euphorbiaceae; Salic $=$ Salicaceae; Sapot $=$ Sapotaceae $;$ Rub $=$ Rubiaceae; Melas $=$ Melastomataceae; Apoc $=$ Apocynaceae $;$ Voch $=$ Vochysiaceae; Olac $=$ Olacaceae $;$ Clus $=$ Clusiaceae $;$ Anac $=$ Anacardiaceae.$*$ Famílias que dentro de uma área estudada não fizeram parte das mais ricas.

Table 3 - Number of species per family among the richest family in the forests of the Chapada Diamantina, Bahia, Brazil. The number in parentheses refers to the order of family's predominance studies. 1. Present study, 2. Montane forests in the Bacia mountain chain, 3. Ciliary forests along the Lençóis River; 4. Ciliary forests along the Capivara River; 5. Ciliary forests Ribeirão River; 6. Ciliary forests along the Mandassaia River; 7. Montane forests besides of the Lençóis River Valley; 8. Montane forests besides of the Capivara River Valley; 9. Plateau forest; 10. Crevice forest; 11. Montane forest. * = Families within of study area were not part of the richest.

\begin{tabular}{lllllllllllllll}
\hline Área & Fonte & Myrt & Lau & Fab & Chrys & Euph & Sapot & Rub & Melas & Apoc & Voch & Clus & Anac \\
\hline 1 & Presente estudo & $9(1)$ & $6(2)$ & $6(2)$ & $4(3)$ & $4(3)$ & $3(4)$ & $*$ & $2(5)$ & $*$ & $*$ & $*$ & $*$ \\
2 & Souza (2007) & $6(1)$ & $2(5)$ & $4(3)$ & $2(4)$ & $6(1)$ & $*$ & $5(2)$ & $*$ & $*$ & $*$ & $*$ & $*$ \\
3 & Funch (1997) & $14(1)$ & $*$ & $10(2)$ & $4(4)$ & $5(3)$ & $3(5)$ & $*$ & $5(3)$ & $3(5)$ & $3(5)$ & $*$ & $*$ \\
4 & Stradmann(2000) & $5(2)$ & $*$ & $8(1)$ & $2(5)$ & $4(3)$ & $4(3)$ & $*$ & $2(5)$ & $*$ & $3(4)$ & $*$ & $*$ \\
5 & Stradmann(1997) & $6(1)$ & $2(3)$ & $2(3)$ & $2(3)$ & $*$ & $2(3)$ & $*$ & $3(2)$ & $*$ & $*$ & $3(2)$ & $2(3)$ \\
6 & Ribeiro-Filho & $4(1)$ & $*$ & $*$ & $*$ & $2(3)$ & $*$ & $2(3)$ & $*$ & $3(2)$ & $*$ & $4(1)$ & $2(3)$ \\
& et al. (2009) & & & & & & & & & & & & \\
7 & Funch (1997) & $5(2)$ & $*$ & $8(1)$ & $3(4)$ & $4(3)$ & $2(5)$ & $2(5)$ & $3(4)$ & $3(4)$ & $*$ & $*$ & $*$ \\
8 & Stradmann(2000) & $*$ & $2(3)$ & $6(1)$ & $3(2)$ & $3(2)$ & $3(2)$ & $*$ & $*$ & $2(3)$ & $2(3)$ & $*$ & $*$ \\
9 & Funch 2008 & $6(1)$ & $*$ & $6(1)$ & $3(3)$ & $4(2)$ & $3(3)$ & $*$ & $2(4)$ & $2(4)$ & $*$ & $*$ & $*$ \\
10 & Funch 2008 & $6(1)$ & $*$ & $2(2)$ & $*$ & $*$ & $*$ & $2(2)$ & $*$ & $*$ & $*$ & $*$ & $2(2)$ \\
11 & Nascimento 2009 & $20(1)$ & $10(2)$ & $4(5)$ & $*$ & $4(5)$ & $3(8)$ & $4(5)$ & $5(3)$ & $3(8)$ & $3(8)$ & $3(8)$ & $*$ \\
\hline
\end{tabular}


Tabela 4-Constância relativa (CR) das famílias e gêneros de maior riqueza específica nas diferentes formações florestais da Chapada Diamantina, Bahia, Brasil.

Table 4 - Relative Constancy (CR) of the families and genera of the richest families in different forest formations of the Chapada Diamantina, Bahia, Brazil.

\begin{tabular}{llll}
\hline Família & CR $^{(\%)}$ & Gênero & CR $^{(\%)}$ \\
\hline Anacardiaceae & 100 & Calyptranthes & 100 \\
Apocynaceae & 100 & Pouteria & 100 \\
Leguminosae & 100 & Simarouba & 100 \\
Myrtaceae & 100 & Tapirira & 100 \\
Sapotaceae & 100 & Clusia & 91 \\
Simaroubaceae & 100 & Miconia & 91 \\
Clusiaceae & 91 & Myrcia & 91 \\
Euphorbiaceae & 91 & Byrsonima & 82 \\
Melastomataceae & 91 & Guapira & 82 \\
Burseraceae & 82 & Protium & 82 \\
Chrysobalanaceae & 82 & Aspidosperma & 73 \\
Malpighiaceae & 82 & Heisteria & 73 \\
Nyctaginaceae & 82 & Himatanthus & 73 \\
Rutaceae & 82 & Inga & 73 \\
\hline
\end{tabular}

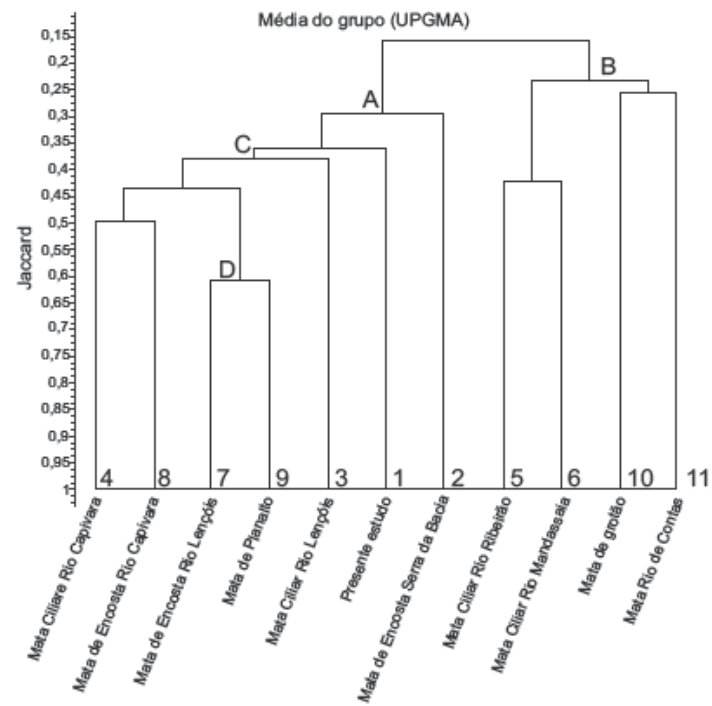

Figura 3 - Dendrograma de similaridade de Jaccard e UPGMA, incluindo a área de estudo e mais dez áreas de florestas na Chapada Diamantina, Bahia, Brasil. As áreas estão numeradas conforme apresentada na Tabela 1.

Figure 3 - Dendrogram of Jaccard similarity and UPGMA, including the study area and ten forest areas in Chapada Diamantina, Bahia, Brazil are number as shown in Table 1.

\section{Discussão}

Em geral, as florestas da borda oriental da Chapada Diamantina apresentam uma estratificação horizontal semelhante: o dossel é contínuo com árvores que variam de 8 a $12 \mathrm{~m}$ representadas por Tapirira guianensis, Clusia nemorosa, Alchornea triplinervia, Balizia pedicellaris, Diospyros sericea, Bowdichia virgilioides, Maprounea guianensis, Emmotum nitens e Vochysia pyramidalis; e emergentes que atingem $20 \mathrm{~m}$ como Aspidosperma discolor e Hymenolobium janeirense var. stipulatum (Funch et al. 2002, 2008).

Nas florestas da borda ocidental as árvores do dossel apresentam altura entre 6 a $9 \mathrm{~m}$, com emergentes atingindo até $35 \mathrm{~m}$, como Pouteria torta (Nascimento 2009). No presente estudo, verificou-se a presença de dossel mais elevado, atingindo $16 \mathrm{~m}$ de altura e com representantes recorrentes em outras florestas além de Micropholis gardneriana, Pogonophora schomburgkiana e Pouteria ramiflora, e emergentes com cerca de $26 \mathrm{~m}$, assim como observado por Funch et al. $(2002,2008)$ para as FES (florestas de planalto). Verifica-se que as florestas estacionais submontanas (florestas de planalto) sobre latossolo na borda leste da Chapada Diamantina apresentam o estrato vegetacional mais alto que as demais florestas.

O sub-bosque da floresta analisada apresenta elevado número de jovens das espécies dos estratos superiores, sugerindo que esta comunidade se encontra em crescimento, por ser constituída em sua maioria de jovens, com eficiente regeneração (Marangon 1999). Outro elemento abundante na floresta são as trepadeiras que, segundo Udulutsch et al. (2004), são importantes no funcionamento das florestas estacionais por fornecem recursos à fauna, aspecto também observado nas outras áreas florestais da Chapada (Souza 2007, Funch et al. 2008).

Considerando o estrato arbóreo, encontrouse poucas famílias, totalizando mais da metade das espécies observadas. A concentração de riqueza específica em poucas famílias está de acordo com a maioria dos estudos em florestas estacionais semideciduais realizados na Chapada Diamantina (Funch 1997; Stradmann 2000), bem como em outras áreas do Nordeste (Rodal et al. 2005; Neves 2005) e Sudeste (Leitão-Filho 1987; Ivanauskas et al. 1999; Santos \& Kinoshita 2003; Yamamoto et al. 2005; Marangon et al. 2003; Souza et al. 2003). 
Fabaceae, Euphorbiaceae, Myrtaceae, Rubiaceae e Lauraceae se destacaram no presente estudo e nas outras dez áreas estudadas na Chapada Diamantina (Tab. 3), estando entre as famílias com alta constância e alta riqueza nas florestas estacionais semideciduais do Brasil (Oliveira-Filho \& Fontes 2000; Santos \& Kinoshita 2003) e entre as famílias mais ricas em espécies lenhosas do mundo (Yamamoto et al. 2005). Myrtaceae é a família com o maior número de espécies na Chapada Diamantina, tanto na borda oriental quanto na borda ocidental, seguida por Fabaceae, Chrysobalanaceae, Sapotaceae, Melastomataceae e Apocynaceae.

Juntamente com as famílias anteriormente citadas, Anacardiaceae e Simaroubaceae apresentam maior constância relativa (Tab. 4). Essas famílias são de grande importância ecológica, com elevada riqueza florística em diversos ecossistemas (Funch 1997; Oliveira-Filho \& Fluminhan-Filho 1999). Estes dados corroboram com a hipótese de Yamamoto et al. (2005) de que se uma família é muito rica em espécies e tem uma distribuição muito ampla, ela constará dentre as famílias de maior constância e maior riqueza em qualquer lugar de sua área de distribuição.

Embora a composição de famílias encontrada seja a esperada para as florestas estacionais, o presente estudo acrescentou muito à riqueza de espécies (88) das FESS da borda leste da Chapada Diamantina: estudos prévios registraram apenas 45 espécies, distribuídas em 21 gêneros e 25 famílias (Funch et al. 2005, 2008). Portanto, há necessidade de mais estudos nos fragmentos de florestas estacionais do estado da Bahia, em especial na Chapada Diamantina, como já evidenciado por Cardoso \& Queiroz (2008) e Cardoso et al. (2009), os quais registraram novas ocorrências para a Bahia, além de coletas raras, em fragmentos de florestas estacionais.

Neste sentido, este levantamento amostrou 18 espécies arbóreas ainda não registradas para quaisquer tipos florestais para a Chapada Diamantina, como Guatteria oligocarpa (Annonaceae), Cordia bicolor (Boraginaceae), Swartzia bahiensis (Fabaceae) e Nectandra membranaceae (Lauraceae). Além disso, este estudo reforçou os resultados de Funch et al. (2008), que ao considerar o conjunto florístico de espécies arbóreas presentes nas formações florestais da Chapada Diamantina, verificou que apenas Eschweilera tetrapetala (Lecythidaceae) se apresenta como espécie restrita às FESS (florestas de planalto), estando fortemente associada à presença de latossolo.

Os resultados da análise de similaridade florística entre as florestas da Chapada Diamantina revelou maior similaridade florística do componente arbóreo entre as áreas 7 e 9 (38 espécies em comum), entre as áreas 4 e 8 (31 espécies em comum) e entre o presente estudo e os grupos C e A (41 espécies em comum). O conjunto de espécies das áreas 4, 7, 8 e 9 compõe formações florestais que se situam em cotas altitudinais semelhantes, entre 500 e $700 \mathrm{~m}$, em geral apresentando episódios notáveis de queda foliar na estação seca.

A dissimilaridade do grupo A com o grupo B deve-se provavelmente às particularidades ambientais apresentadas por este último, o que reflete em algumas espécies exclusivas como Clusia melchiorii e Guapira obtusata. São florestas perenifólias acima de $800 \mathrm{~m}$ de altitude com fornecimento hídrico, seja fluvial ou por chuvas orográficas. As florestas 5 e 6 estão localizadas a margem de rios. A floresta 10 (mata de grotão) situase em fendas (falhas geológicas) relativamente estreitas na encosta das serras, proporcionando ambientes sombreados e protegidos do fogo e ocupada por espécies restritas a ambientes úmidos como Hedyosmum brasiliense Mart. ex Miq., Clethra scabra Pers. e Drimys brasiliensis Miers. (Guedes \& Orge 1998; Neves 2005; Funch et al. 2008); e a floresta 11 apresenta-se com constante neblina que contribui para o aumento da umidade.

É válido ressaltar que o índice de similaridade de Jaccard que une o grupo B (25\%) é baixo quando comparado com o grupo $\mathrm{D}(60 \%)$, o que demonstra acentuada heterogeneidade florística entre as florestas. Considerando o tamanho continental do Brasil, as florestas da Chapada Diamantina são próximas geograficamente, mas florestas próximas podem apresentar composição florística distinta, dependendo das condições ambientais predominantes em cada uma delas (Ribeiro-Filho et al. 2009).

A Chapada Diamantina ocupa aproximadamente $15 \%$ do território baiano. No entanto estudos florísticos sobre seus ecossistemas florestais, principalmente estacionais, ainda são escassos, especialmente a região situada entre o norte de Minas Gerais e o centro-sul da Bahia da Cadeia do Espinhaço. Tal fato limita um melhor entendimento das relações florísticas sobre as florestas dessas regiões. 
O presente estudo contribuiu com o registro de 57 espécies não catalogadas para as FESS do Parque Nacional da Chapada Diamantina (Tab. 2). Das 88 espécies arbóreas inventariadas, 70 são recorrentes em florestas estacionais da Chapada, sendo 18 ainda não registradas para quaisquer tipos de florestas, o que reforça a necessidade de ampliação dos estudos florísticos e estruturais para melhor revelar a sua riqueza e heterogeneidade. Ressalta-se a ocorrência da espécie Eschweilera tetrapetala, a única espécie apontada como restrita para as FESS da Chapada Diamantina, corroborando estudos prévios de Funch et al. (2005, 2008).

\section{Agradecimentos}

Os autores agradecem ao $\mathrm{CNPq}$ a concessão da bolsa de mestrado ao primeiro autor, à FAPESB o apoio financeiro para realização do projeto, ao IBAMA a concessão da licença de pesquisa, ao apoio do Programa de Pós-Graduação em Botânica da UEFS, a R. Funch, M.A. Santos e N.F.B. Cruz a ajuda no trabalho de campo, a Fundação Chapada Diamantina a hospedagem durante toda pesquisa, às identificações dos taxonomistas: A. Rapini (Apocynaceae), S.C. Ferreira (Asteraceae), M.M.S. Castro (Bignoniaceae), M.N.S. Statf (Boraginaceae), D.S.C. Torres (Euphorbiaceae), L.P. Queiroz (Fabaceae), P.D. Carvalho (Malpighiaceae), A.K.A. Santos e R. Goldenberg (Melastomataceae), R.B. Santos e L.S. Funch (Myrtaceae), C. Van den Berg (Orchidaceae), R.P. Oliveira (Poaceae), E.B.M. Silva (Polygalaceae), J.G. Jardim (Rubiaceae), além de J.R.S. Silva, F. França, E. Melo, e F.H.Nascimento.

\section{Referências}

APG - The Angiosperm Phylogeny Group. 2003. An update of the Angiosperm Phylogeny Group classification for the orders and families of flowering plants: APG II. Botanical Journal of the Linnean Society 141:399-436.

Cardoso, D.B.O.S. \& Queiroz, L.P. 2008. Floristic composition of seasonally dry tropical forest fragments in Central Bahia, Northeastern Brazil. Journal of the Botanical Research Institute of Texas 2: 551-573.

Cardoso, D.B.O.S.; França, F.; Novais, J.S.; Ferreira, M.H.S.; Santos, R.M.; Carneiro, V.M.S. \& Gonçalves, J.M. 2009. Composição florística e análise fitogeográfica de uma floresta semidecídua na Bahia, Brasil. Rodriguésia 60: 1055-1076.

Couto, A.P.L. 2008. Composição florística e estrutura de uma floresta estacional submontana, Parque Nacional da Chapada Diamantina, Lençóis, Bahia, Brasil. Dissertação de Mestrado. Universidade Estadual de Feira de Santana, Feira de Santana. 51p.
Diegues, A.C. \& Arruda, R.S.V. 2001. Saberes tradicionais e biodiversidade no Brasil. Biodiversidade Vol. 4. Ministério do Meio Ambiente, Brasília; USP, São Paulo. 176p.

Franca Rocha, W.J.S.; Juncá, F.A.; Chaves; J.M. Funch, L.S. 2005. Considerações finais e recomendações para conservação. In: Juncá, F.A.; Funch, L. \& Franc Rocha, W.J.S. (ed.). Biodiversidade e conservação da Chapada Diamantina. Ministério do Meio Ambiente, Brasília. Pp. 409-435.

Ferraz, E.M.N.; Araújo, E.L. \& Silva, S.I. 2004. Floristic similarities between lowland and montane areas of Atlantic Coastal Forest in Northeastern Brazil. Plant Ecology 174: 59-70.

Funch, L.S. 1997. Composição florística e fenologia de mata ciliar e mata de encosta adjacentes ao rio Lençóis, Lençóis, Bahia. Tese de Doutorado. UNICAMP, Campinas. 298p.

Funch, L.S. 2008. Florestas da região norte do Parque Nacional da Chapada Diamantina e seu entorno. In: Funch L.S.; Funch, R.R. \& Queiroz L.P. Serra do Sincorá - Parque Nacional da Chapada Diamantina. Ed. Radam, Feira de Santana. Pp. 63-77.

Funch, L.S.; Funch, R.R. \& Barroso, G.M. 2002. Phenology of gallery and montane forest in the Chapada Diamantina, Bahia, Brazil. Biotropica 34: 40-50.

Funch, L.S.; Funch, R.R.; Harley, R.; Giulietti, A.M.; Queiroz, L.P.; França, F.; Melo, E.; Gonçalves, C.N. \& Santos, T. 2005. Florestas estacionais semideciduais. In: Juncá, F.A.; Funch, L. \& Rocha, W. (ed.). Biodiversidade e conservação da Chapada Diamantina. Ministério do Meio Ambiente, Brasília. Pp. 181-193.

Funch, L.S.; Rodal, M.J.N. \& Funch, R.R. 2008. Floristic aspects of the forests of the Chapada Diamantina, Bahia, Brazil. In: Thomas, W. \& Briton, E.G. (org.). The Atlantic Coastal Forest of Northeastern Brazil. Mem. of the New York Botanical Garden Press 100: 193-220.

Funch, R.R.; Harley, R.M. \& Funch, L.S. 2009. Mapping and evaluation of the state of conservation of the vegetation in and surrounding the Chapada Diamantina National Park, ne Brazil. Biota Neotropica 9: 11-12.

Guedes, M.L.S \& Orge, M.D.R. 1998. Checklist das espécies vasculares do morro do Pai Inácio (Palmeiras) e Serra da Chapadinha (Lençóis), Chapada Diamantina, Bahia, Brasil. EDUFBA, Salvador. 68p.

Harley. R.M. 1995. Introdução. In: Stannard, B.L. (ed.). Flora of the Pico das Almas, Chapada Diamantina, Bahia, Brasil. Royal Botanic Gardens, Kew. Pp. 43-78.

Ivanauskas, N.M.; Rodrigues, R.R. \& Nave, A.G. 1999. Fitossociologia de um trecho de floresta estacional semidecidual em Itatinga, São Paulo, Brasil. Scientia Forestalis 56: 83-99.

Ivanauskas, N.M.; Monteiro, R.; Rodrigues, R.R. 2000. Similaridade florística entre áreas de Floresta Atlântica 
no estado de São Paulo. Brazilian Journal of Ecology 1-2: 71-81.

Jesus, E.F.; Falk, F.H. \& Marques, T.M. 1985. Caracterização geográfica e aspectos geológicos da Chapada Diamantina, Bahia. Centro Editorial e Didático da Bahia, Salvador. 50p.

Kamino, L.H.Y.; Oliveira-Filho, A.T. \& Stehmann, J.R. 2008. Relações florísticas entre as fitofisionomias florestais da Cadeia do Espinhaço, Brasil. In: Megadiversidade Cadeia do Espinhaço: avaliação do conhecimento científico e prioridade de conservação. Vol. 4, no 1-2. Conservação Internacional. Pp. 39-77.

Kershaw, K.A. \& Looney, J.H.H. 1985. Quantitative and dynamic plant ecology. Edward Arnold, London. 282p.

Leitão-Filho, H.F. 1987. Considerações sobre a florística de florestas tropicais e sub-tropicais do Brasil. IPEF 35: 41-46.

Leitão Filho, H. F. 1992. A flora arbórea da Serra do Japi. In: Morellato, L.P.C. (org.). História natural da Serra do Japi: Ecologia e preservação de uma área florestal no sudeste do Brasil. Editora da Unicamp/ Fapesp, Campinas, Pp. 40-62.

Marangon, L.C. 1999. Florística e fitossociologia de área de floresta estacional semidecidual visando dinâmica de espécies florestais arbóreas no município de Viçosa, MG. Tese de Doutorado. Universidade Federal de São Carlos, São Carlos. 135p.

Marangon, L.C.; Soares, J.J. \& Feliciano, A.L.P. 2003. Florística arbórea da Mata da Pedreira, município de Viçosa, Minas Gerais. Revista Árvore 27: 207-215.

Meira-Neto, J.A.A.; Bernacci, L.C.; Grombone, M.T.; Tamashiro, J.Y. \& Leitão-Filho, H.F. 1989. Composição florística da floresta semidecídua de altitude do Parque Municipal da Grota Funda (Atibaia, estado de São Paulo). Acta Botanica Brasilica 3: 51-74.

Morellato, L.P.C. \& Haddad, C.F.B. 2000. Introduction: The Brazilian atlantic forest. Biotropica 32: 786-792.

Mori, S.A.; Silva, L.A.M.; Lisboa, G. \& Coradin, L. 1989. Manual de manejo do herbário fanerogâmico. CEPEC/CEPLAC, Ilhéus. 104p.

Müeller-Dombois, D. \& Ellenberg, H. 1974. Aims and methods of vegetation ecology. New York, Wiley.

Nascimento, F.H.F. 2009. As florestas alto montanas nordestinas, sul da Chapada Diamantina, Bahia: florística, estrutura e relações biogeográficas. Tese de Doutorado. Universidade Estadual de Feira de Santana, Feira de Santana. 250p.

Neves, M.L.C. 2005. Caracterização da vegetação de um trecho de mata atlântica de encosta na Serra da Jibóia, Bahia. Dissertação de Mestrado. Universidade Estadual de Feira de Santana, Feira de Santana. 101p.

Oliveira, R.J. 2006. Variação da composição florística e da diversidade alfa das florestas atlânticas no estado de São Paulo. Tese de Doutorado. Universidade Estadual de Campinas, Campinas. 144p.

Oliveira-Filho, A.T.; Vilela, E.A.; Gavilanes, M.L. \& Carvalho, D.A. 1994. Comparison of the woody flora and soils of six areas of montane semideciduous forest in southern Minas Gerais, Brazil. Edinburgh Journal of Botany 51: 355- 389.

Oliveira-Filho, A.T. \& Fluminhan-Filho, M. 1999. Ecologia da vegetação do Parque Florestal Quedas do Rio Bonito. Cerne 5: 52-64.

Oliveira-Filho, A.T. \& Fontes, M. A. 2000. Patterns of floristic differentiation among Atlantic forests in Southeastern Brazil and the influence of climate. Biotropica 32: 793-810.

Oliveira-Filho, A.T.; Jarenkow, J.A. \& Rodal, M.J.N. 2005a. Floristic relationships of seasonally dry forests of eastern South America based on tree species distribution patterns. In: Pennington, R.T.; Lewis, G.P. \& Ratter, J.A. (orgs.). Neotropical savannas and dry forests: plant diversity, biogeography, and conservation. CRC Press, Boca Raton. Pp. 151-184.

Oliveira-Filho, A.T.; Tameirão-Neto, E.; Carvalho, W.A.C.; Werneck, M.; Brina, A.E.; Vidal, C.V.; Rezende, S.C. \& Pereira, J.A.A. 2005b. Análise florística do compartimento arbóreo de áreas de floresta atlântica sensu lato na região das bacias do leste (Bahia, Minas Gerais, Espírito Santo e Rio de Janeiro). Rodriguésia 56: 185-235.

Peixoto, G.L.; Martins, S.V.; Silva, A.F.; \& Silva, E. 2005. Estrutura do componente arbóreo de um trecho de floresta atlântica na Área de Proteção Ambiental da Serra da Capoeira Grande, Rio de Janeiro, RJ, Brasil. Acta Botanica Brasilica. 19: 539-547.

RADAMBRASIL. 1981. Levantamento de recursos naturais. Folha SD.24 - Salvador. Geologia, geomorfologia, pedologia, vegetação e uso potencial da terra. Ministério das Minas e Energia, Rio de Janeiro. 624p.

Ribeiro-Filho, A.A.; Funch, L.S. \& Rodal. M.J.N. 2009. Composição florística da Floresta Ciliar do Rio Mandassaia, Parque Nacional da Chapada Diamantina, Bahia, Brasil. Rodriguésia 60: 265-276

Ribeiro, J.E.L.S.; Hopkins, M.J.G.; Vicentini, A.; Sotheres, C.A.; Costa, M.A.S.; Brito, J.M.; Souza, M.A.; Martins, L.H.P.; Lohmann, L.G.; Assunção, P.A.C.L.; Pereira, E.C.; Silva, C.F.; Mesquita, M.R. \& Procópio, L.C. 1999. Flora da Reserva Ducke: Guia de identificação das plantas vasculares de uma floresta da terra-firme na Amazônia Central. INPA, Manaus. 800p.

Rizzini, C.T. 1997. Tratado de fitogeografia de Brasil: aspectos ecológicos, sociológicos e florísticos. Âmbito Cultural, Rio de Janeiro. 747p.

Rodal, M.J.N.; Lucena, M.F.A.; Andrade, K.V.S.A. \& Melo, A.L. 2005. Mata do Toró: uma floresta estacional semidecidual de terras baixas no nordeste do Brasil. Hoehnea 32: 283-294. 
Santos, K. \& Kinoshita, L.S. 2003. Flora arbustivoarbórea do fragmento de floresta estacional semidecidual do Ribeirão Cachoeira, município de Campinas, SP. Acta Botanica Brasilica 17: 325-341.

Salis, S.M.; Shepherd, G.J. \& Joly, C.A. 1995. Floristic comparison of mesophytic semi- deciduous forests of the interior of the state of São Paulo, southeast Brazil. Vegetatio 119: 155-164.

Scudeller, V.V.; Martins, F.R. \& Shepherd G.J. 2001. Distribution and abundance of arboreal species in the atlantic ombrophilous dense forest in Southeastern Brazil. Plant Ecology 152: 185-199.

SEIA. Sistema Estadual de Informações Ambientais da Bahia. 2010. Unidades de Conservação - APA Marimbus/Iraquara. Disponível em <http:// www.seia.ba.gov.br/seuc/unidades $>$. Acesso em 17 out 2010.

Siqueira, M.F. 1994. Análise florística e ordenação de espécies arbóreas da Mata Atlântica através de dados binários. Dissertação de Mestrado. Universidade Estadual de Campinas, Campinas. 143p.

Sousa, A.E.A. 2007. Composição florística e estrutura de uma mata de encosta, Serra da Bacia, Parque Nacional da Chapada Diamantina, Palmeiras, Bahia, Brasil. Dissertação de Mestrado. Universidade Estadual de Feira de Santana, Feira de Santana.

Souza, J.S.; Espírito-Santo, F.D.B.; Fontes, M.A.L.; Oliveira-Filho, A.T. \& Botezelli. L. 2003. Análise das variações florísticas e estruturais da comunidade arbórea de um fragmento de floresta semidecídua às margens do Rio Capivari, Lavras - MG Revista Árvore 27: 185-206.

Stradmann, M.T.S. 1997. Composição florística de um trecho de mata ciliar da Trilha do Brotão e estudo quantitativo do estrato arbóreo-arbustivo. Rio Ribeirão Parque Nacional a Chapada Diamantina, Bahia - Brasil. Monografia de conclusão de curso. UFBA, Salvador.

Stradmann, M.T.S. 2000. Composição florística da mata ciliar da foz do Rio Capivara e análise quantitativo do estrato arbustivo-arbóreo. Rio Ribeirão Parque Nacional a Chapada Diamantina. Dissertação de Mestrado. Universidade Federal da Bahia, Salvador. 130p.

Torres, R.B.; Martins, F.R. \& Gouvêa, L.S.K. 1997. Climate, soil and tree flora relationships in forests in the state of São Paulo, southeastern Brazil. Revista Brasileira de Botânica 20: 41-49.

Veloso, H.P.; Rangel-Filho, A.L.R. \& Lima, J.C.A. 1991. Classificação da vegetação brasileira adaptada a um sistema universal. IBGE, São Paulo.

Veloso, H.P.; Rangel-Filho, A.L.R. \& Lima, J.C.A. 1992. Manual técnico da vegetação brasileira. IBGE, Rio de Janeiro. 92p.

Udulutsch, R.G.; Assis, M.A. \& Picchi, D.G. 2004. Florística de trepadeiras numa floresta estacional semidecídua, Rio Claro - Araras, estado de São Paulo, Brasil. Revista Brasileira de Botânica 27: 125-134.

Yamamoto, L.F.; Kinoshita, L.S. \& Martins, F.R. 2005. Florística dos componentes arbóreo e arbustivo de um trecho da Floresta Estacional Semidecídua Montana, município de Pedreira, estado de São Paulo. Revista Brasileira de Botânica 28: 191-202. 